\title{
A new C-band SAR for ERS-1 underflights
}

\section{Madsen, Søren Nørvang; Skou, Niels; Christensen, Erik Lintz}

\section{Published in:}

Record of the IEEE International Radar Conference

Link to article, DOI:

10.1109/RADAR.1990.201166

Publication date:

1990

Document Version

Publisher's PDF, also known as Version of record

Link back to DTU Orbit

Citation (APA):

Madsen, S. N., Skou, N., \& Christensen, E. L. (1990). A new C-band SAR for ERS-1 underflights. In Record of the IEEE International Radar Conference (pp. 223-227). IEEE. https://doi.org/10.1109/RADAR.1990.201166

\section{General rights}

Copyright and moral rights for the publications made accessible in the public portal are retained by the authors and/or other copyright owners and it is a condition of accessing publications that users recognise and abide by the legal requirements associated with these rights.

- Users may download and print one copy of any publication from the public portal for the purpose of private study or research.

- You may not further distribute the material or use it for any profit-making activity or commercial gain

- You may freely distribute the URL identifying the publication in the public portal 


\section{A NEW C-BAND SAR FOR ERS-1 UNDERFLIGHTS}

S. Nørvang Madsen, N. Skou, and E. Lintz Christensen Electromagnetics Institute, Technical University of Denmark

\section{ABSTRACT}

Since 1986 the Electromagnetics Institute, the Technical University of Denmark, has designed, build and tested a high resolution airborne C-band Synthetic Aperture Radar, SAR. The first test flights took place in November and December 1989. The radar design has been based on digital technology to the largest possible extent, to make the system as adaptable as possible. This has resulted in a very flexible radar with variable resolution, swathwidth, and imaging geometry to mention just a few of the more important features. Special attention was paid to system calibration when the system was designed. This paper will outline design considerations, and discuss selected features in relation to the implementation (for instance: the digital system, the aircraft, the antenna and its installation as well as the calibration system), and the first test results will be shown. Finally the on-going system developments and project plans are briefly described.

\section{INTRODUCTION}

The principle of Synthetic Aperture Radar was "invented" nearly 40 years ago and the first unclassified papers were published 20 years ago [1]. For many years the subject was mainly of military interest first of all due to the fact that civilian applications could not justify the development of the costly SAR systems. Therefore the development of SARs focused on the military needs such as high resolution and long range. Today the potential benefits of SAR systems are much more widely accepted, and a number of new SARs has been developed for research but also for operational applications as for instance sea ice surveillance.

The KRAS ( = Coherent Radar and Advanced Signal processing) system described in this paper is a research radar which:

1) serves as a testbed for coherent radar technology

2) provides a facility that is well suited for underflights in relation to the SAR of the European ERS-1 satellite.

\section{KRAS DESIGN}

The top level parameters that must be determined first when designing a SAR system are: frequency, polarization, resolution, swathwidth, sensitivity, range, and operating geometry. The KRAS frequency and polarization were primarily given by the fact that the system should be compatible with and applicable to underflights of the European ERS-1 SAR satellite, [2], now scheduled for launch in 1991. The KRAS radar is accordingly a single frequency system operating at 5.3 $\mathrm{GHz}$. The polarization is presently VV, but funding has been obtained to start the development of a dual-polarized antenna.The ERS-1 satellite SAR acquires data at steep angles of incidence, it is therefore important that the aircraft SAR operates at very high altitudes, since the ground swath corresponding to a given incidence angle interval will be proportional to altitude. At an early stage of the design it was realized that the best suited altitude levels would be above
$30000 \mathrm{ft}$.

The potential applications of the KRAS SAR spans a wide range from geophysical mapping, sea ice monitoring, and fishery inspection, all typically requiring a wide swath though with moderate resolution requirements (i.e. $10 \mathrm{~m}$ ), to cartography mapping and reconnaissance, where a high resolution is more important than a wide swath. The radar is designed to achieve a resolution down to $2 \mathrm{~m}$ by $2 \mathrm{~m}$. The nominal range of the system is $80 \mathrm{~km}$ and the effective slant range swathwidth after pulse-compression is between 9 and $48 \mathrm{~km}$ depending on the resolution and transmitted pulselength. The swath is basically limited by the number of complex range cells, which is 8192, except at the steepest angles of incidence where the antenna elevation beamwidth of $30^{\circ}$ will limit the useful swath. The range off-set is programmable. Three typical mapping set-ups are shown in figure 1 .

The system sensitivity corresponds to $10 \mathrm{~dB}$ SNR for distributed targets with a characteristic backscatter coefficient of $\sigma^{\circ}=-30 \mathrm{~dB}\left(\mathrm{~m}^{2} / \mathrm{m}^{2}\right)$ at an incidence angle of $\Theta=65^{\circ}$ (altitude $12.5 \mathrm{~km}$ slant-range $=30 \mathrm{~km}$ ), and a $10 \mathrm{~dB}$ SNR for a point target with $\sigma=-10 \mathrm{~dB}\left(\mathrm{~m}^{2}\right)$ at a range of $80 \mathrm{~km}$. Both assumes an azimuth sample spacing of $0.1875 \mathrm{~cm}$.

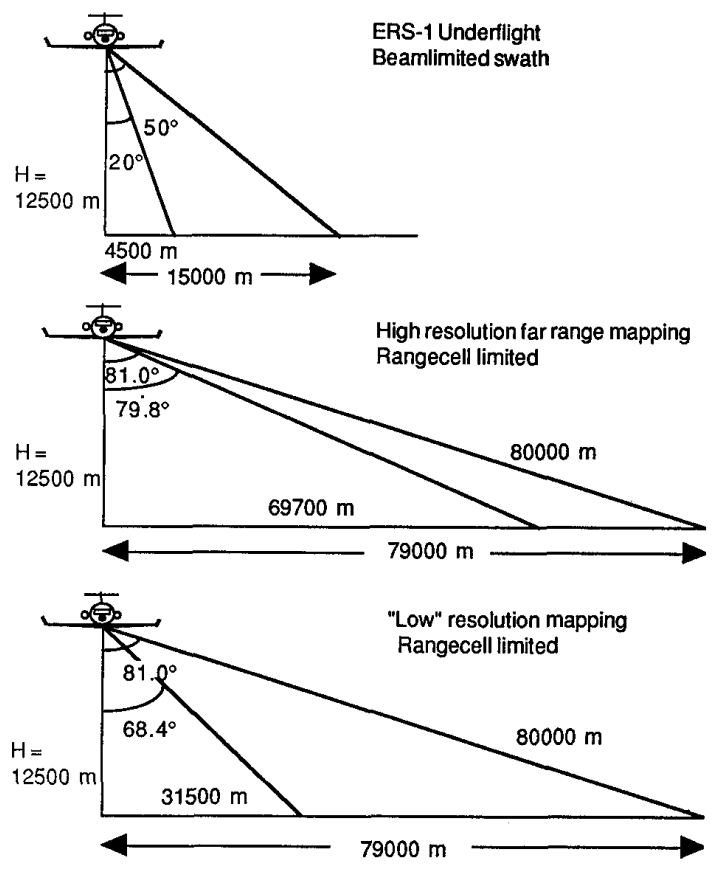

Figure 1. Examples showing some possible mapping configurations for the KRAS SAR.

CH2882-9/90/0000-0223\$1.00 (c) (1990 IEEE)

IEEE INTERNATIONAL RADAR CONFERENCE 


\section{SYSTEM IMPLEMENTATION}

Digital system: Obviously significant flexibility is required to meet the requirements. The variable resolution and swath can only be obtained if signals of varying bandwidth can be transmitted and the processing in the receiver can be modified accordingly. A trade-off analysis considering multiple SAW devices, a swept-oscillator system and a digital signal generation scheme showed that only the digital system could provide the desired range of pulse durations, bandwidths and coding algorithms in a cost effective way. The application of digital pulse compression in the receiver gives a number of advantages though at the expense of quite heavy processing requirements. Also, compared to the dynamic range of the $\mathrm{AD}$ converters themselves, the dynamic range (measured as the ratio between the largest point target and the noise floor) after digital compression is improved by the processing gain.

There are a number of ways to generate the digital codes and similarly there are several ways to transform the digital numbers to analog signals. The most simple approach is to apply direct phase modulation on the RF carrier. Such a system is very attractive when phase codes with few differen phasors and moderate sidelobes are required. This coding process, however, introduces phase steps whose effect must be included in the design [3]. Alternatively one can use DA converters to generate the modulation function at video frequencies, either using one DA-converter and applying a digital frequency off-set (which means that to generate signals of bandwidth $B$ the sampling rate must be larger than 2 B), or by using a quadrature modulator as shown in figure 2 . Since the quadrature modulator generates the complex modulation function $m(t)=a(t) \exp (j \phi(t))$ at baseband (covering frequencies from $-B / 2$ to $+B / 2$ ), each $D A$ converter needs only sample at a rate larger than $B$. The latter implementation is well suited for high bandwidth signals and was therefore chosen for the KRAS signal generator.

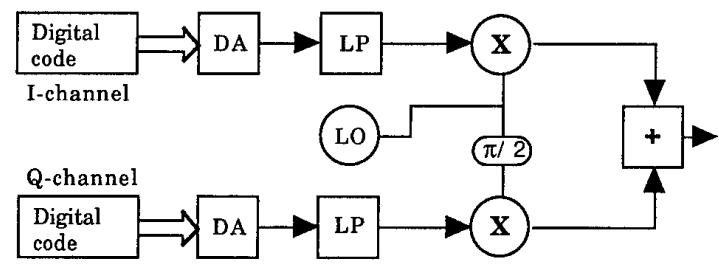

Figure 2. Digital signal generator implemented as a quadrature baseband modulator.

Also the digital codes can be generated in a number of ways. One method to generate the quadratic phase of a linear FM is for instance to implement a digital double integration of a constant. Alternatively the pulse code can be generated off-line and down loaded in a fast buffer memory. This approach gives significantly more flexibility and the software generating the codes can be designed to calculate non-linear FM with specified pulse length, bandwidth and sidelobe level. The price paid for this flexibility is that a fairly large and very fast buffer memory is required. It is important to note that high quality signals with low sidelobes can only be obtained if: the gains of the quadrature channels are equal, the channels are properly time aligned, and the phase difference between the channels is $90^{\circ}$. The KRAS digital signal generator is based on a quadrature modulator with the converters running at a $200 \mathrm{MHz}$ sampling rate. The low-pass filters have a cut-off frequency of $150 \mathrm{MHz}$ and a nearly linear phase characteristic. 4096 complex code words are stored in a RAM buffer, hence supporting pulses of a length up to $20 \mu \mathrm{s}$. The codes are generated by a C-program that supports both linear and nonlinear FM codes. The incorporation of signal pre-distortion is presently being studied, thereby enabling correction of system imperfections, [4]. Preliminary results have shown that when Hamming weighting is applied (nominal PSLR $=-42 \mathrm{~dB}$ and ISLR $=-27 \mathrm{~dB}$ ) the system sidelobe peak-side-lobes are $33 \mathrm{~dB}$ suppressed when pre-distortion is not applied, and $41 \mathrm{~dB}$ suppressed when pre-distortion is exploited.

Aircraft: It was found that the Gulfstream G-3 aircraft (owned and operated by the Royal Danish Air Force) is an excellent platform for a SAR intended for satellite underflights. It is a very stable platform that can operate at high altitudes (up to $45.000 \mathrm{ft}$ ) and the nominal airspeed of $240 \mathrm{~m} / \mathrm{s}$ reduce the motion compensation problems relative to slower aircraft. Due to the present configuration of the aircraft the antenna installation was placed under the fuselage near the wings, see figure 3 . To enable fast mounting and dismounting the antenna system was placed in a modified fighter-plane fuel tank.

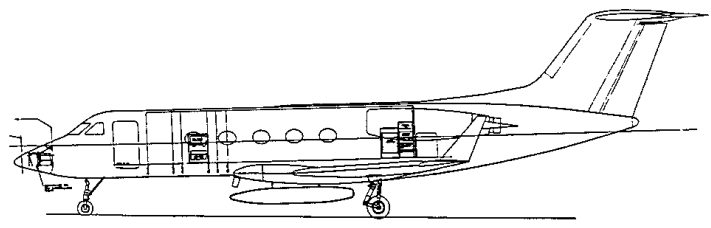

Figure 3. Gulfstream G-3 with the KRAS SAR pod

Antenna. Originally the elevation pattern was planned to be a modified cosec square pattern $\left(\mathrm{G}(\psi) \sim \operatorname{cosec}^{2} \psi \sqrt{\cos \psi}\right)$ ). However, it was found that with the limited height of the antenna $(27 \mathrm{~cm})$ it was not possible to achieve the cosec squared pattern without significant ripple on the elevation pattern, and furthermore a sufficient beam stability over the full bandwidth was not obtained. Therefore the modified cosec square pattern had to be more "modified" than originally planned. The slotted waveguide antenna was calibrated in the Electromagnetics Institutes Radio Anechoic Chamber using spherical near field antenna measurement techniques. Measurements have revealed elevation sidelobes of $-28 \mathrm{~dB}$ at the centre frequency.

Antenna installation: The antenna is mounted on a ball joint close to the antennas phase centre. The antenna is stabilized in roll, pitch and yaw. The antenna installation raised two problems: 1) How to minimize the effect of the radome, 2) how to avoid interference fringes due to multipath propagation via the wing. It is found that depending on the look angle of the antenna, the incidence angle of the radiated field

\section{IEEE INTERNATIONAL RADAR CONFERENCE}


relative to the radome is between $0^{\circ}$ and $50^{\circ}$ (worst case). By using a so-called A-sandwich which consists of a layer of fibre glass, a core of low $\varepsilon$ material, and another fibre glass layer (skin thickness of $0.025 \lambda$, core thickness $0.2 \lambda$ ) one finds that for the VV polarization the transmittivity is larger than $98 \%$ for incidence angles up to $60^{\circ}$. Also, the insertion phase delay is constant for incidence angles up to $60^{\circ}$. If the transmission loss of $2 \%$ is assumed to be due to reflections (which is a reasonable assumption at the rather low frequency of 5 $\mathrm{GHz}$ ) this corresponds to $\rho=-17 \mathrm{~dB}$. This was verified by measurements of the sky temperature by a $5 \mathrm{GHz}$ radiometer with and without a sample of the radome material in front of the antenna. These measurements showed $\rho$ values between 17 and $-20 \mathrm{~dB}$ for angles up to $30^{\circ}$ (maximum range for setup). The second concern was the influence of the wing. Although only the front edge of the wing is in the view of the antenna, two-way propagation and the accompanying interference problem has been a major concern. A maximum twoway gain/loss of $1 \mathrm{~dB}$ (theoretical worst case assuming the wing is a perfect and flat reflector) gave rise to an elevation sidelobe specification of $-25 \mathrm{~dB}$.

Motion compensation: The motion compensation system of the KRAS radar is considered one of the critical subsystems. Similar to the accuracy requirements for a real antenna, the individual elements of a synthetic antenna must be located with an accuracy corresponding to fractions of a wavelength. The investigation of the motion compensation problem is extremely complex and it involves questions like: Requirements for the position and attitude measurement, achievement of sufficient measurement accuracy, implementation of a real time algorithm. In the system implemented, an INU (Inertial Navigation Unit) is mounted in the antenna pod (to minimize the lever arm correction). To allow for correction of INU velocity drift the SAR includes a real-time Doppler tracker unit, and the SAR processor is presently being upgraded to include an autofocus module. The motion compensation system uses a Kalman filter system to find the nominal flight track during a $5 \mathrm{~km}$ alignment track and after this the mapping starts and the nominal track is essentially locked. A more detailed paper on the motion compensation system is planned.

The KRAS parameters are listed in Table 1 and the system block diagram is shown in figure 6 .

\begin{tabular}{|c|c|}
\hline Frequency: & $5.3 \mathrm{GHz}$ \\
\hline Transmitter peak power: & $2 \mathrm{~kW}$ \\
\hline Receiver noise figure: & $2.5 \mathrm{~dB}$ \\
\hline System loss (estimate): & $3 \mathrm{~dB}$ \\
\hline Pulse length: & from 0.64 to $20 \mu \mathrm{s}$ \\
\hline Maximum bandwidth: & $100 \mathrm{MHz}$ \\
\hline \multirow{4}{*}{$\begin{array}{l}\text { Antenna gain: } \\
\text { azimuth 3-dB beamwidth: } \\
\text { elevation pattern width: } \\
\text { polarization: }\end{array}$} & $26.8 \mathrm{~dB}$ \\
\hline & $2.7^{\circ}$ \\
\hline & $30^{\circ}$ \\
\hline & lVV \\
\hline Resolution range: & Variable $2,4,8 \mathrm{~m}$ \\
\hline azimuth: & Variable $2,4,8 \mathrm{~m}$ \\
\hline Slant range mapping width (raw data): & Variable $12,24,48 \mathrm{~km}$ \\
\hline Range: & $80 \mathrm{~km}$ \\
\hline
\end{tabular}

Table 1: KRAS system parameters

\section{SYSTEM CALIBRATION}

Calibration is an important issue in modern remote sensing radars. Several precautions are taken in the KRAS radar design to facilitate system calibration. First of all the stability of the system is ensured by applying design techniques usually applied in radiometer systems. One of the important factors is that all RF components, including the TWT, are temperature stabilized. Another important feature of radiometers is that of frequent automatic calibration. This is implemented in the KRAS radar design by implementing a number of internal calibration loops. Both a sample of the TWT drive signal and the TWT high power output ( sampled via a $-60 \mathrm{~dB}$ cross coupler) are injected into the calibration switch. The calibration switch includes a programmable precision attenuator and an average power monitor, and the sample of the drive signal or the attenuated high power signal can be injected in the receiver either before or after the low noise amplifier.

Use of corner reflectors and comparison between the collected SAR data and a C-band noise scatterometer that was recently developed at the Electromagnetics Institute, will also contribute to the calibration of the system.

\section{FLIGHT TESTS}

In November and December, 1989, the first flight tests of the Danish airborne SAR were performed. Already on its first mission it produced valuable data with a resolution of 8 by $8 \mathrm{~m}$. Motion compensation was not applied. In the second mission Copenhagen and the Technical University of Denmark were mapped.

To assist the planning of the missions a number of plots showing the received power as a function of altitude, look-angle and ground range have been plotted. The plots are based on the measured antenna pattern and a $0.375 \mathrm{~m}$ azimuth pulse spacing. The noise floor using a $100 \mathrm{MHz}$ bandwidth is at $-91.5 \mathrm{dBm}$. The datatake covering the Technical University of Denmark and central Copenhagen were at an altitude of $41000 \mathrm{ft}(\approx 12500 \mathrm{~m})$, at slant ranges from $22000 \mathrm{~m}$ to $31200 \mathrm{~m}$ and using a look-angle of $65^{\circ}$. The signal transmitted was an $80 \mathrm{MHz}$ linear FM. From figure 4 it is seen that the power returned for a given $\sigma^{\circ}$ will vary approximately $1.5 \mathrm{~dB}$ from near-range to far-range.

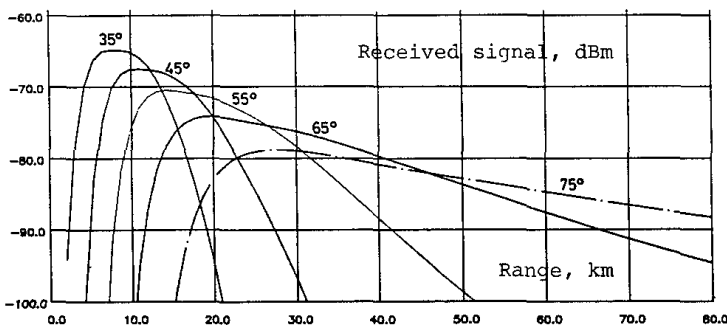

Figure 4. Plot showing the return power from a "standard" target with $\sigma^{\circ}=-20 \mathrm{~dB}$ (with look angle as parameter).

In figure 5 is shown a radar image of central Copenhagen. Motion compensation was applied, and a resolution of 2 by $2 \mathrm{~m}$ was verified by analysis of three corner reflector located at the Technical University of Denmark. The data shown 


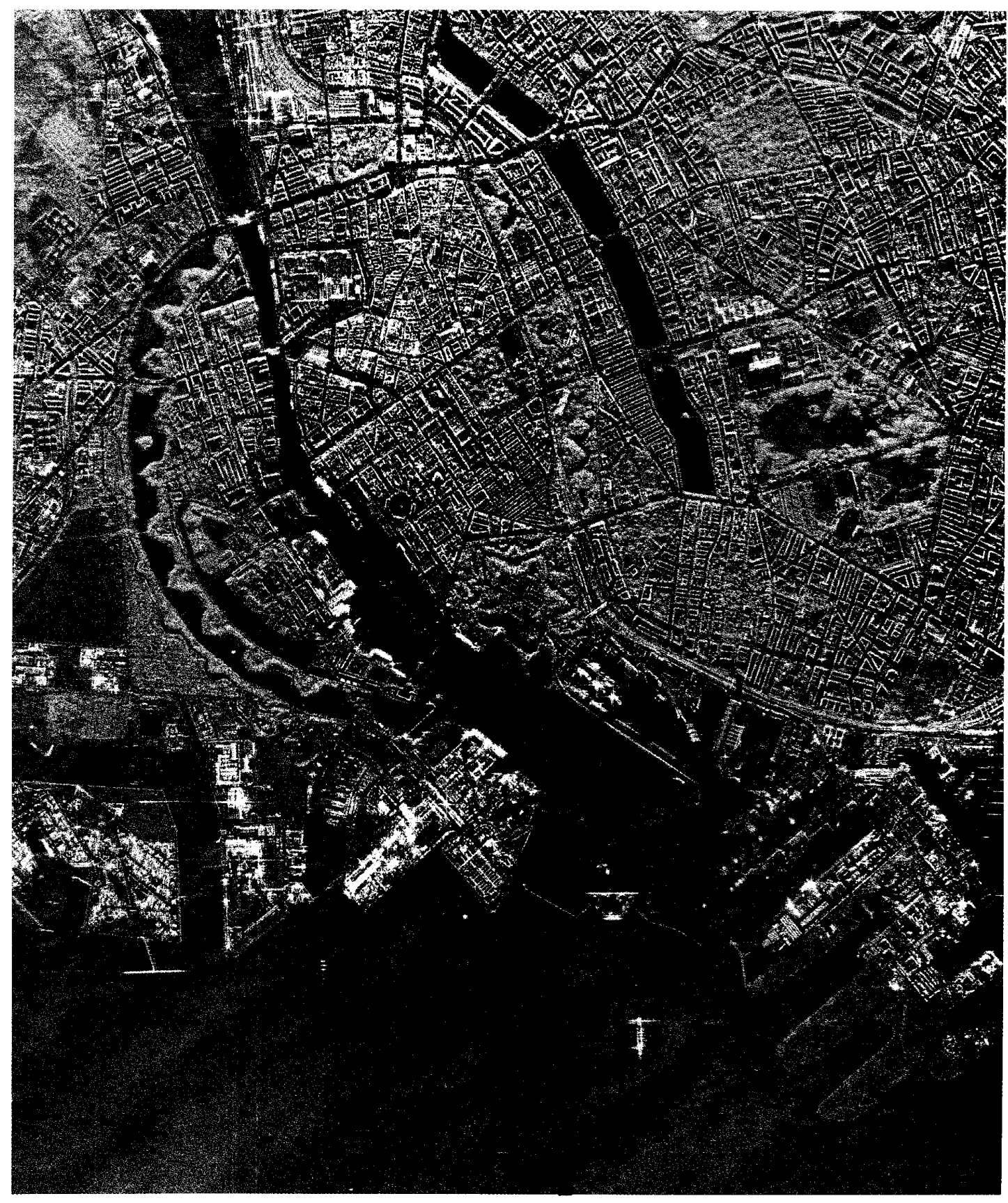

Figure 5. C-band SAR image of Copenhagen showing the city center and the harbour from $25 \mathrm{~km}$ distance. The image width is $6 \mathrm{~km}$ and the resolution is 2 by 2 meters.

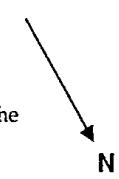

IEEE INTERNATIONAL RADAR CONFERENCE 


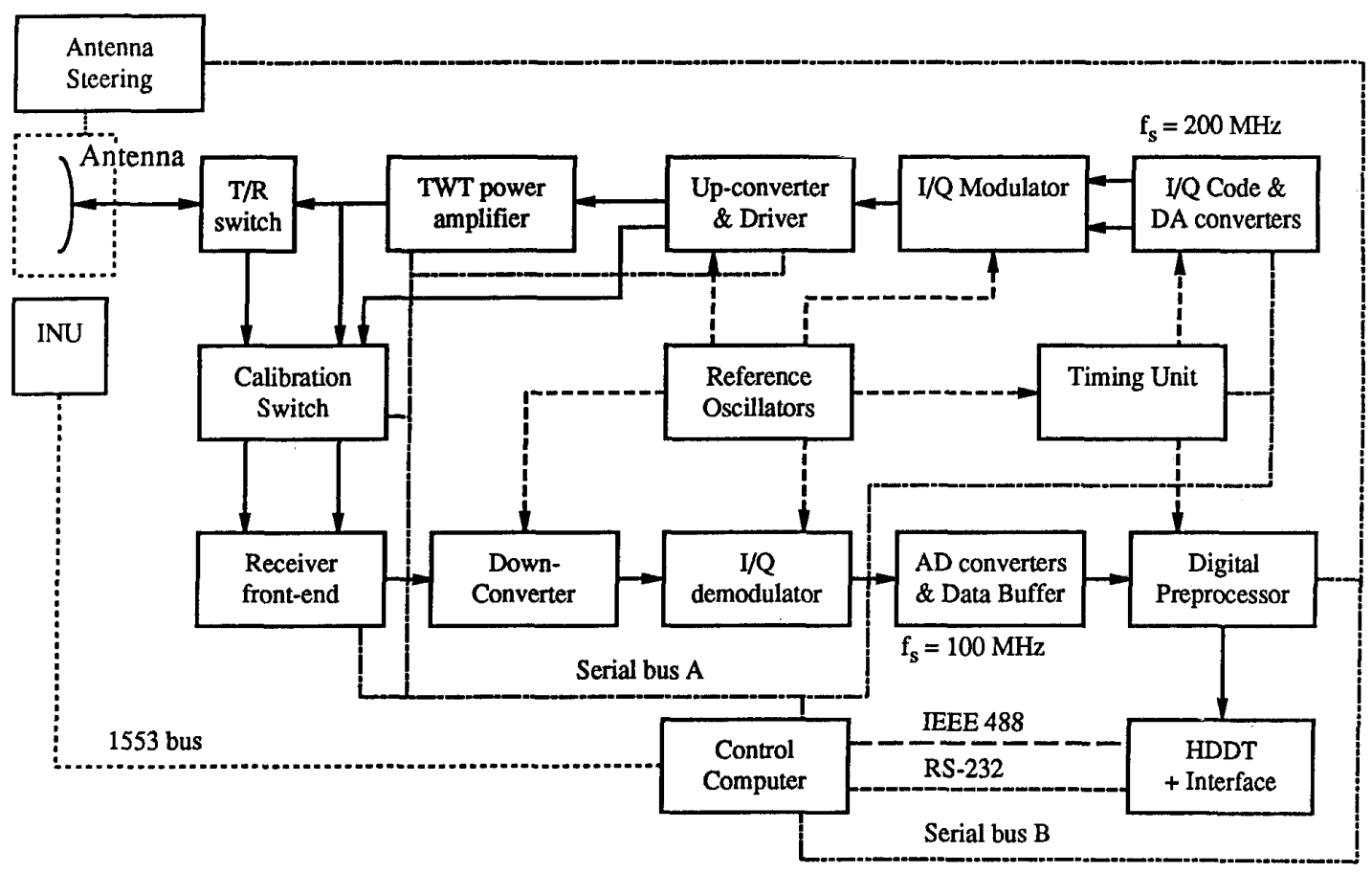

Figure 6. Block diagram of the KRAS system

has not been radiometrically equalized and STC (sensitivity time control) was not applied during recording. The equalization of the radiometric response across the swath is entirely due to the antenna shaping. It is noted that there are no interference fringes indicating reflections from the wing, and there are no "banding" effect in the along track direction indicating motion compensation problems.

\section{FUTURE PLANS}

At the moment the system is being tested in more detail. This is expected to result in modifications that will make the system more easy to operate. It is expected that a second generation of the system software will be available in the spring 1990. These tests will also contribute to the calibration of the system.

The development of a real-time processor and display system is also underway. It is expected that the processor will be available by the end of 1991 or early 1992. Recently funding was approved for developing a prototype of a dual-polarized microstrip antenna This work has just started, and it is hoped that it will lead to an up-date of the system to full polarimetric capability.

\section{REFERENCES}

[1] Kovaly John J. Synthetic Aperture Radar. Artech House, 1976

[2] G. Duchossois Overview and Status of the ERS-1 Programme ESA SP-254, Vol.1., August 1986

[3] Iglehart, S.C Some Results on Digital Chirp IEEE trans. on Aerospace and Electronic Systems, Vol. AES-14, No. 1 pp 118-127, 1978

[4] Netterstrøm A

Digital Correction of Analog Signal Processing Errors Int. conf. on Radar 89, 24-28 April, 1989, Paris, Vol 1, pp 313-318

[5] Vincent ].D.

Radomes

In: Skolnik "Radar Handbook", Mcgraw-Hill, 1970

\section{AUTHORS' MAILING ADDRESS}

Electromagnetics Institute, Technical University of Denmark Building 348, Lundtoftevej 100, DK 2800 Lyngby, Denmark Phone +45 428814 44, Fax +45 45931634 , Telex 37529 DTHDIA DK 